Classification

Physics Austracts

$61.30-31.20 \mathrm{~N}$

\title{
Theoretical conformational study of the TBBA molecule (*)
}

\author{
H. Perrin and J. Bergès \\ Laboratoire de Physique Moléculaire, Tour 22, Université Pierre et Marie Curie, \\ 4, Place Jussieu, 75230 Paris Cedex 05, France \\ and U.E.R. d'Etudes Médicales et Biologiques, Université René Descartes, Paris, France
}

(Reçu le 29 mars 1982, accepté le 15 juin 1982)

\begin{abstract}
Résumé. - L'analyse conformationnelle de la molécule de TBBA a été effectuée à l'aide de la méthode PCILO. Les résultats ont permis de définir les zones de basse énergie et d'en déduire les conformations les plus stables du TBBA. Le rôle respectif du cœur et des chaînes dans les variations des sections moléculaires est discuté.
\end{abstract}

\begin{abstract}
A conformational analysis of the TBBA molecule has been performed using a quantic semi-empirical method : PCILO. The results allow us to define the low energy zones and to deduce the most stable conformations of the TBBA molecule. The respective contributions of aromatic core and terminal chains to the variations of the molecular area is discussed.
\end{abstract}

1. Introduction - The TBBA molecule, which has the particularity of showing a great polymorphism in its liquid crystal phases, has been the subject of many experimental studies (magnetic resonance of deuterated molecules [1-3] and X-ray diffraction [4] in particular). These studies have emphasized the role of internal distortions. For this reason we present here the conformational analysis of the TBBA molecule : we wish to specify, from an energetic point of view, the possibilities of motions in the aromatic core as well as in the terminal butyl chains. Some recent papers $[5,6,7]$ have emphasized the interest of conformational calculations to study mesogen compounds.

We have studied the isolated molecule, and we shall concentrate on the extent of the low energy zones (inside which the energetic minimum should lie whatever be the phase), and on the heights of the potential barriers rather on the position of an absolute minimum.

2. Results of the calculations. - We have used a semi-empirical quantic method, the PCILO $\left({ }^{1}\right)$ [8] one : this is a perturbational method, in which the wavefunction is built from orbitals localized on chemical bonds. This last point is somehow open to criticism, in the case of highly conjugated molecules, like the ones we are studying here, since the double bonds must be also localized. In a preliminary work [9], we have shown that determining the energy by calculating the arithmetic mean of the energies of the different Kekule formulas leads to satisfying results. Besides, we use here this method in preference to the INDO $\left({ }^{2}\right)$ one, because the results which have been formerly

$\left(^{*}\right)$ La version française de cet article est proposée pour publication aux Comptes Rendus de l'Académie des Sciences.

( $\left.{ }^{1}\right)$ PCILO : Perturbational Configuration Interaction of Localized Orbitals.

$\left({ }^{2}\right)$ INDO : Intermediate Neglect of Differential Overlap. 
obtained on the BA molecule $[9,10]$ with the PCILO method were in better agreement with the experimental results.

We have separated the conformational study of the aromatic core from that of the terminal chains, since after having done some calculations on the whole TBBA molecule, we noted that the conformational changes of the core had no influence on the conformational changes of the chains and vice-versa.

We thus present successively the results for the TBA molecule (Terephtal-di-aniline) and the butyl-benzene one.

2.1 TBA MOLECULE (AROMATIC CORE). - In a preliminary paper we presented the complete conformational analysis of the BA molecule and we have determined the geometry of the azomethin group after energy optimization, using PCILO method. We kept this geometry for the TBA molecule.

$$
\begin{gathered}
r_{\Phi-\mathrm{N}}=1.41 \AA, \quad r_{\mathrm{N}=\mathrm{C}}=1.30 \AA, \quad r_{\mathrm{C}-\Phi}=1.46 \AA \\
\Phi-\mathrm{C}-\mathrm{N}=122^{\circ}, \quad \mathrm{C}-\mathrm{N}=\mathrm{C}=120^{\circ}, \quad \mathrm{N}=\mathrm{C}-\mathrm{C}=123^{\circ}, \quad \mathrm{C}-\mathrm{C}-\Phi=122^{\circ} .
\end{gathered}
$$

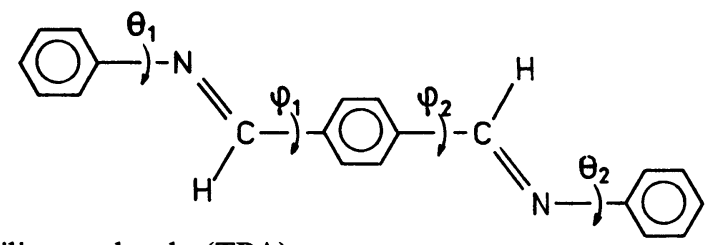

Fig. 1. - Terephtal-di-aniline molecule (TBA).

The results obtained for the BA molecule can be generalized to the TBA molecule. Indeed the $E=f\left(\theta_{1}\right)$ and $E=f\left(\theta_{2}\right)$ curves for TBA are identical to the $E=f(\theta)$ [9] one for BA, and similarly for $E=f\left(\phi_{1}\right)$ and $E=f\left(\phi_{2}\right)$.
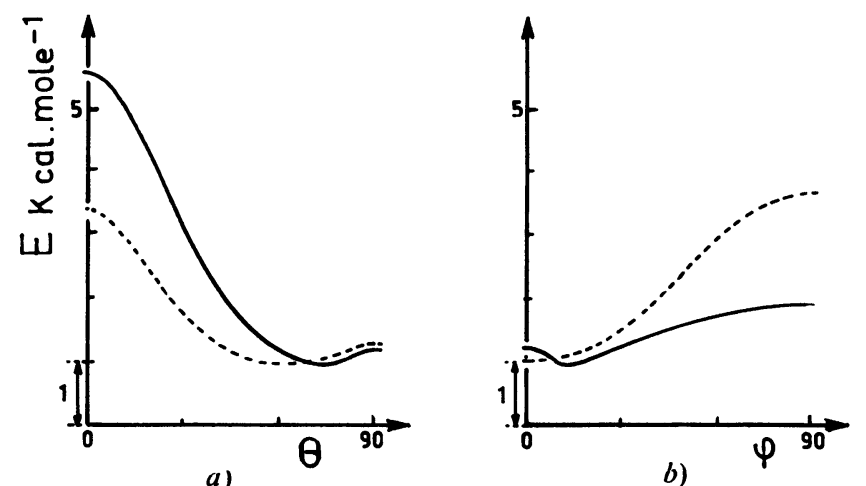

Fig. 2. - Energy of the TBA molecule. a) $E=f\left(\theta_{1}\right)=f\left(\theta_{2}\right)$ for $\phi_{1}=\phi_{2}=0^{\circ}$. b) $E=f\left(\phi_{1}\right)=f\left(\phi_{2}\right)$ for $\theta_{1}=\theta_{2}=60^{\circ}$.

However, in the isolated molecule a large number of conformations are energetically equivalent because of the independence of the rotations around the different bonds and of the symmetries existing inside the molecule. Our calculations do not allow us to differentiate some conformations (those where $\phi_{2}=\phi_{1}$ from those where $\phi_{2}=-\phi_{1}, \phi_{2}=\Pi+\phi_{1}$ and $\phi_{2}=\Pi-\phi_{1}$, as well as those where $\theta_{2}=\theta_{1}$ from those with $\theta_{2}=-\theta_{1}$ ). 
It is only by introducing the intermolecular forces that one could be able to distinguish between them.

We find that the values of $\theta$ and $\phi$ which lead to the lowest energies lie between $50^{\circ}$ and $130^{\circ}$ for $\theta_{1}$ and $\theta_{2}$, and $-30^{\circ}$ and $+30^{\circ}$ for $\phi_{1}$ and $\phi_{2}$. From this we obtain that the angle between the plane of the central ring and the plane of a terminal ring lies between $20^{\circ}$ and $80^{\circ}$. This result is consistent with the values obtained by DMR [1], i.e. $60^{\circ}$ in smectic phase and $80^{\circ}$ in nematic phase. On the other hand, in the crystalline phase [4], there are different values for the angles between the plane of the central ring and that of one terminal ring $\left(52.9^{\circ}\right)$ and between the plane of the central ring and that of the other terminal ring $\left(16.1^{\circ}\right)$.

The first value is in agreement with our results, but the second one is slightly outside the interval we have determined, that clearly demonstrates the role of intermolecular forces.

2.2 The BUTYL-BENZENE MOLECUle. - The energy is only slightly dependent on the geometry of the butyl chain. The energy curves are very similar, when using the standard parameters $\left(r_{\mathrm{CC}}=1.54 \AA, r_{\mathrm{CH}}=1.09 \AA, \mathrm{CCC}=109.5^{\circ}, \mathrm{CCH}=109.5^{\circ}\right)$ as well as when using the experimental ones [11] $\left(r_{\mathrm{CC}}=1.54 \AA, \mathrm{CCC}=112.9^{\circ}, \mathrm{CCH}=110.2^{\circ}\right)$. We have only optimized the angle $\omega$ and the length $r$ because they lie close to the ring. We have found $\omega=120^{\circ}$ and $r=1.48 \AA$.

Fig. 3. - Butyl-benzene molecule.

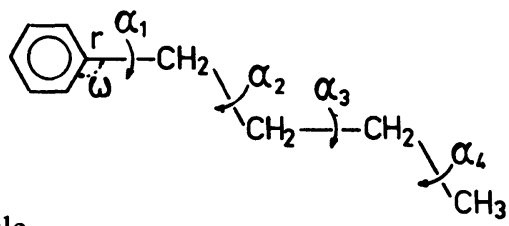

As regards the rotations around the bonds we started from the preferential conformations of a free butyl chain, and from there we examined the modifications due to the presence of the benzene ring. The most perturbated rotation being certainly $\alpha_{1}$ we first consider its variations for values of $\alpha_{2}$ and $\alpha_{3}$ corresponding to the most stable positions of a free butyl chain i.e. : trans-trans $(\mathrm{t}-\mathrm{t})$, trans-gauche $(\mathrm{t}-\mathrm{g})$, gauche-trans $(\mathrm{g}-\mathrm{t})$ and gauche-gauche $(\mathrm{g}-\mathrm{g})$. Once the minimum energy region has been determined for $\alpha_{1}$, we shall examine the variations of $\alpha_{2}$ and $\alpha_{3}$, in order to see what modifications the presence of the ring brings. It did not seem necessary to us to vary $\alpha_{4}$, considering its distance to the ring and the results obtained for $\alpha_{2}$ and $\alpha_{3}$.

- Angle $\alpha_{1}$

In figure 4, we present the variations of $\alpha_{1}$. Note that the minimum lies about $90^{\circ}$, being sometimes shifted towards $100^{\circ}$. The height of barrier depends only on $\alpha_{2}:$ it is about $1.5 \mathrm{kcal}$./mole if $\alpha_{2}$ is in trans position, whereas it is very high if $\alpha_{2}$ is in gauche position. The value of $90^{\circ}$ found for the minimum which indicates that the butyl chain is clearly out of the plane of the ring, is in agreement with the value obtained by Doucet et al. [4] in the crystalline phase and with the hypothesis of Deloche [2] and Dianoux et al. in DMR [1].

- Angles $\alpha_{2}$ and $\alpha_{3}$

After having fixed $\alpha_{1}$ to $90^{\circ}$, we studied the variations of $\alpha_{2}$ and $\alpha_{3}$. The energy map, $E=f\left(\alpha_{2}, \alpha_{3}\right)$ is presented in figure 5 .

The following points can be noted :

- When the chain is in relatively extended conformations (both $\alpha_{2}$ and $\alpha_{3}$ lying between $120^{\circ}$ and $240^{\circ}$ ) the behaviour is very much like in a free butyl chain.

- When the chain is folded, the presence of the ring leads to important restrictions and there is no stable position for values of $\alpha_{2}$ and $\alpha_{3}$ which are both either smaller than $60^{\circ}$ or greater than 


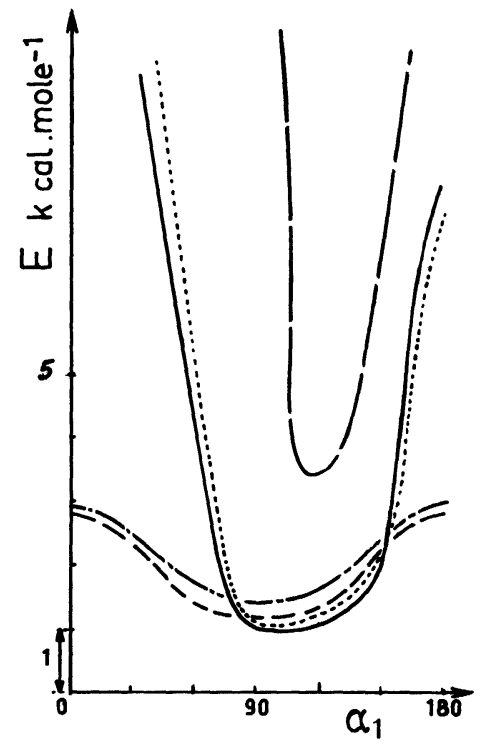

Fig. 4. - Energy of the butyl-benzene molecule. $E=f\left(\alpha_{1}\right)$, for $\alpha_{2}, \alpha_{3}:-\cdots-\mathrm{t}$, t; $\mathrm{g}^{+}, \mathrm{t} ;-\mathrm{g}^{+}, \mathrm{g}^{+} ;----\mathrm{g}^{+}, \mathrm{g}^{-}$.

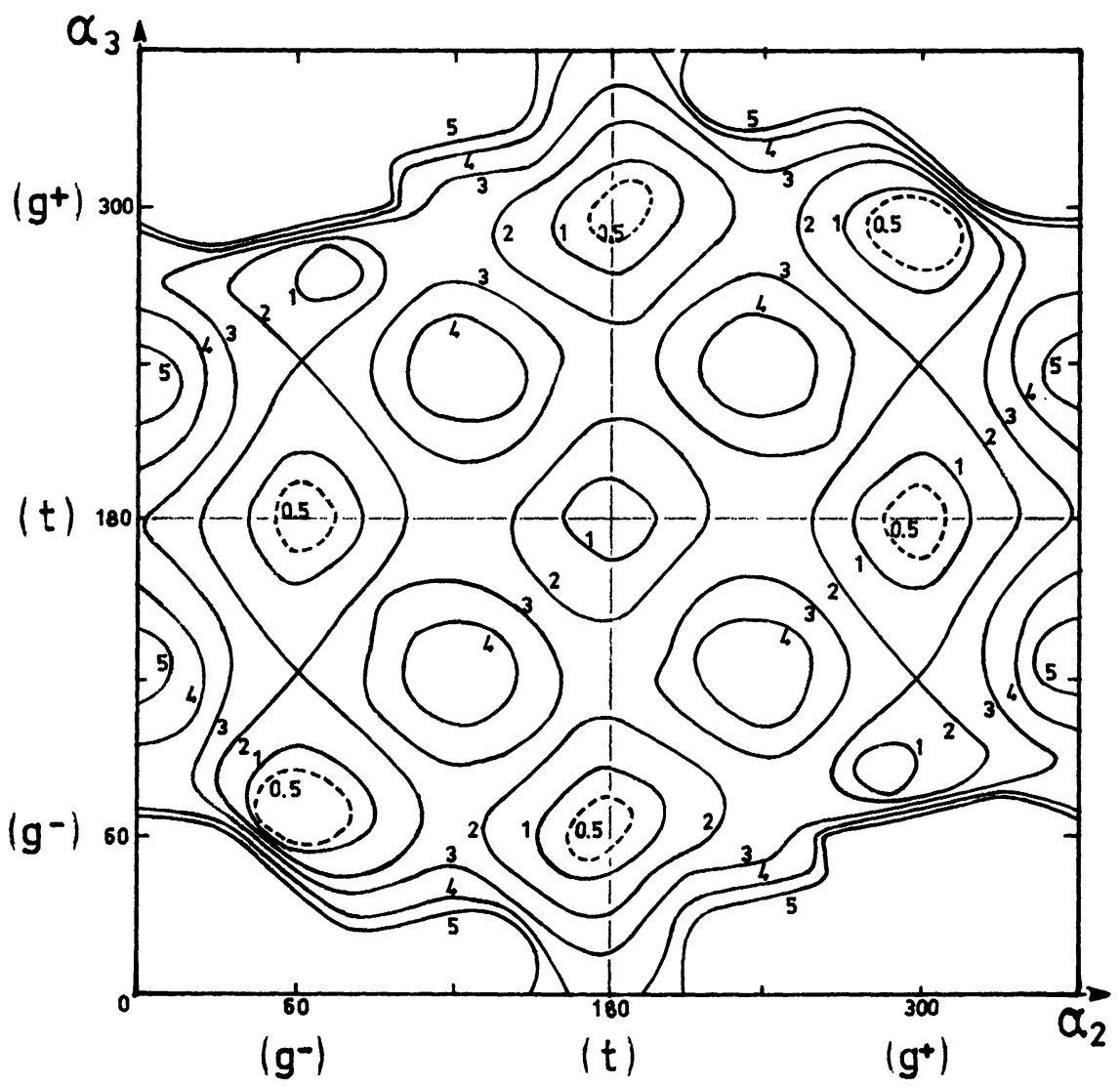

Fig. 5. - Energy of the butyl-benzene molecule. $E=f\left(\alpha_{2}, \alpha_{3}\right)$ for $\alpha_{1}=90^{\circ}$. 
$300^{\circ}$. If there are minima close to these zones they are shifted with respect to a free butyl chain (g-g conformation).

- The constraints are much stronger on $\alpha_{2}$ than on $\alpha_{3}$, as expected, however the absolute minimum occurs for values of $\alpha_{2}$ and $\alpha_{3}$ corresponding to a relatively folded chain, i.e. $\alpha_{2}=60^{\circ}$ $\left(300^{\circ}\right)$ and $\alpha_{3}=75^{\circ}\left(285^{\circ}\right)$ which lie close to a forbidden zone.

- The low energy zone is relatively extended : for the $\mathrm{g}^{+} \mathrm{g}^{+}\left(\right.$or $\left.\mathrm{g}^{-} \mathrm{g}^{-}\right)$conformation, this zone extends from $45^{\circ}$ to $80^{\circ}$ for $\alpha_{2}$ and from $60^{\circ}$ to $80^{\circ}$ for $\alpha_{3}$; for the g-t conformations, it extends from $50^{\circ}$ to $70^{\circ}$ for $\alpha_{2}$ and from $165^{\circ}$ to $195^{\circ}$ for $\alpha_{3}$, for the t-g conformations, it extends from $165^{\circ}$ to $195^{\circ}$ for $\alpha_{2}$ and from $50^{\circ}$ to $70^{\circ}$ for $\alpha_{3}$.

3. Some remarks on the whole TBBA molecule. - As we have mentioned in the introduction the results obtained for the isolated molecule are not sufficient to explain the properties of liquid crystals. It is of course necessary to take into account the interactions between molecules. However these interactions can only bring additional restrictions to the conformations permitted for the isolated molecule. It is likely that in the liquid crystal state and still more in the crystalline state, the molecules will interact in order to lead to an optimal packing. Dilatation measurements [12] have permitted the calculation of the average areas occupied by TBBA in the smectic phase (note that these areas show only small variations in the whole mesomorphic domain). The sections taken perpendicular to the long axis, change from $23 \AA^{2}$ to $25 \AA^{2}$.

We have thus calculated approximative values for the sections of the TBBA molecule for the most stable conformations. We did not try to compare them to experimental values, the calculations being too approximate. Here, we consider the relative variations of the section in a given molecule when its conformation changes.

We find that the variations in the conformations of the aromatic core have little influence : the section varies by $10 \%$ at most. The influence of $\alpha_{1}$ is also negligible. On the contrary, the variations due to the different values of $\alpha_{2}$ and $\alpha_{3}$ are very important since one goes from about $18 \AA^{2}$ for t-g conformations to $23-25 \AA^{2}$ for t-t conformations, to $26-30 \AA^{2}$ for g-g conformations and 30-34 $\AA^{2}$ for g-t ones. Similarly the length of the molecule is 21-23 $\AA$ for g-t and g-g conformations and $26-27 \AA$ for t-t and t-g conformations (the experimental value is $28 \AA$ in crystalline phase and $28.6 \AA$ in smectic phase [4]). In the different conformations of the core and the chains the angle between the para and the long axis of the molecule varies only from $1^{\circ}$ to $9^{\circ}$.

4. Conclusion. - In this study, we tried to examine the conformational properties of the TBBA molecule, which is likely to present a large polymorphism in liquid crystal phases. We have been able to make comparisons between the deformations which may occur in the different parts of the molecule. We have thus established that the possibilities of motions about a stable position are of the same importance in the aromatic cores as in the terminal chains.

On the other hand, the width of the low energy zones in the aromatic core point out its great flexibility whereas the aliphatic terminal chains are more rigid, particularly for the first methylene groups. 


\section{References}

[1] Dianoux, A. J. and Volino, F., J. Physique 41 (1980) 1147.

[2] Deloche, B., Thèse, Université de Paris Sud-Centre d'Orsay (1978).

Charvolin, J. and Deloche, B., J. Physique Colloq. 37 (1976) C3-69.

[3] Boss, P. J. and Doane, J. W., Phys. Rev. Lett. 40 (1978) 1030.

[4] Doucet, J., Thèse, Université de Paris Sud-Centre d'Orsay (1978).

Doucet, J., Mornon, J. P., Chevalier, R. and LifChitz, A., Acta Crystallogr. B 33 (1977) 1701.

[5] Laupretre, F. and Monnerie, L., Eur. Polym. J. 14 (1978) 415.

[6] SY, D. and PTAK, M., J. Physique-Lett. 40 (1979) L-137.

[7] Pesquer, M., Cotrait, M., Marsau, P. and Volpilhac, V., J. Physique 41 (1980) 1039.

Cotrait, M., Marsau, P., Pesquer, M. and Volpilhac, V., J. Physique 43 (1982) 355.

[8] Diner, S., Malrieu, J. P. and Claverie, P., Theor. Chim. Acta 13 (1969) 1.

[9] Bergès, J. and Perrin, H., J. Mol. Struct. Theochem. 76 (1981) 375.

[10] Perrin, H. and Bergès, J., J. Mol. Struct. Theochem. 76 (1981) 299.

[11] Kuchitsu, K., Bull. Chem. Soc. Japan 32 (1959) 748.

[12] Guillon, D. and Skoulios, A., J. Physique 38 (1977) 79. 